\title{
Track insulation verification and measurement
}

\author{
Jacopo Bongiorno ${ }^{1}$, and Andrea Mariscotti 2,* \\ ${ }^{1}$ University of Genova, DITEN, Via Opera Pia 11A, 16145, Genova, Italy \\ ${ }^{2}$ ASTM Sagl, Via Comacini 7, 6830, Chiasso, Switzerland
}

\begin{abstract}
Methods for the measurement of track insulation are reviewed pointing out minimum necessary conditions and practical aspects, as well as consistency and repeatability of results. Track configuration, need of electrical sectioning and use of external electrodes or conductive structure are key parameters to determine suitable methods.
\end{abstract}

\section{Introduction}

Track insulation is a requirement for stray current protection and for functional purposes, if track circuits are used. Contractual limits are often specified referring to EN 50122-2 [1], without clarifying margins during commissioning and the acceptable spread of values for variable conditions and different track sections (drying concrete, pollution, moisture, viaduct, tunnel, defects and variability of the fastening system). This is a common problem to all methods that may be addressed by repeated measurements in different environmental conditions and parts of the system (e.g. at different construction times, concrete settlement and drying).

Many measurement methods exist for track to earth conductance, taken from standards and literature. EN 50122-2 (IEC 62128-2) suggests methods suitable for different track arrangements: continuously welded rails, insulating rail joints including verification of their efficiency, presence of civil structures (tunnels and viaducts). In the following these methods are described, including practical considerations on setup issues derived from site experience. Track section length shall always be accurately measured: a metric wheel should be used; combining the number of sleepers and their gauge (distance between sleepers) is also quite accurate. Target accuracy is in the order of $1 \%$.

A reliable and low-resistance connection to the rail should also be ensured, as for some methods the test current may be very large (in the order of $100 \mathrm{~A}$ ) with the risk of temperature increase and hot spots. Rail clamps and clips are a better solution.

Practical aspects cover necessary logistics and verification of elements extraneous to the measurement itself, influencing results and accuracy: conductive parts touching the rails (e.g. parts of fences and walkways not yet installed), overvoltage protecting devices (OVLDs), substation negative feeder cables, water stagnation, electric noise caused by welding, static converters. etc. For electrically continuous rails all these elements are relevant wherever they occur on the whole line.
Finally, distinction shall always be done between worst cases and average performance (including spread or dispersion), in the perspective of the overall purpose, the evaluation of stray current protection and impact on structures, averaging in realistic exploitation conditions (including to this aim track voltage monitoring).

\section{Measurement methods}

\subsection{EN 50122-2}

EN 50122-2 suggests three methods of rail/track-to-earth conductance measurement depending on track section configuration: with and without a civil structure that represents a continuous conductive reference, for sectioned and electrically continuous tracks.

Common to all methods is the prescription that the measured track section shall be without connections to earth, including substation negative feeders, and Overvoltage Limiting Devices (OVLDs) shall be disconnected. In general, repetition of measurements allows evaluating consistency of results and coping with external transients and disturbance.

\subsubsection{Conductance per length for track sections without civil structure}

This method is suggested for electrically continuous track sections shorter than $2 \mathrm{~km}$ without metallic reinforcement structures.

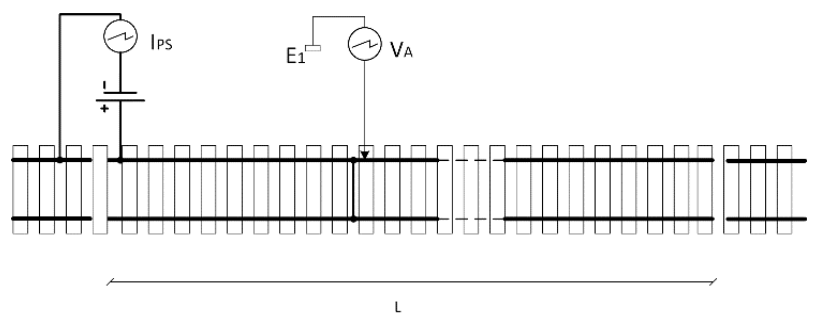

Fig. 1. Track to earth conductance measurement using EN 50122-2 A.3 method.

\footnotetext{
${ }^{*}$ Corresponding author: andrea.mariscotti $@$ astm-e.ch
} 
The section is supplied at one end by a power supply (PS) with positive pole connected to the measured track section and negative pole connected to the tracks behind, used as an earth electrode through their conductance to earth. Track voltage is measured at least $50 \mathrm{~m}$ away from the PS injection point with remote electrode $E_{1}$ as reference, while PS is switched on and off.

Track-to-earth conductance p.u.l. in $\mathrm{S} / \mathrm{km}$ is:

$$
G=\frac{I_{P S}}{L\left(V_{A, o n}-V_{A, o f f}\right)}
$$

where:

$G$ is the track to earth conductance $[\mathrm{S} / \mathrm{km}]$;

$I_{P S}$ is the current of the power supply [A];

$V_{A}$ is the rail-to- $\mathrm{E}_{1}$ voltage [V] for on and off PS;

$L$ is the section length $[\mathrm{km}]$.

Section length has impact on measurement accuracy: if close to the maximum length, the measurement underestimates the real conductance with an error of about $1 \%$ [2]; optimal results are obtained placing the voltage reading near the opposite end with respect to PS.

Usually the PS consists of batteries; current intensity shall guarantee reliable voltage and current readings. Voltages higher than $50 \mathrm{~V}$ are not usually needed and they would turn out in safety issues.

The "behind track section" used for PS negative pole earthing might be not long enough to ensure a low resistance to earth; an electrode may be added to back up PS earthing. Variability of less than $0.1 \%$ was found by simulation in [2] for resistance to earth ranging between 0.1 and $20 \Omega$ ); experimental variability of few $\%$ is reported in [3] for values between 0.5 and $50 \Omega$.

The location of the remote electrode $E_{1}$ is very important. The standard specifies a distance from the track $>30 \mathrm{~m}$, but for high resistivity soils it should be increased and fulfilling of "remote condition" shall be checked (the method in sec. 2.5 may be used).

This method is very sensitive to soil polarization: the PS current changes slightly following an exponential decay; simultaneous measurement of voltage and current is thus important and readings should be taken when the current has reached the plateau (also to reduce curve derivative and local sensitivity, improving uncertainty).

Galvanic insulation is necessary for the channels or the use of separate acquisition systems (synchronization of tens of $\mathrm{ms}$ is sufficient considering polarization dynamics, see Fig. 2).

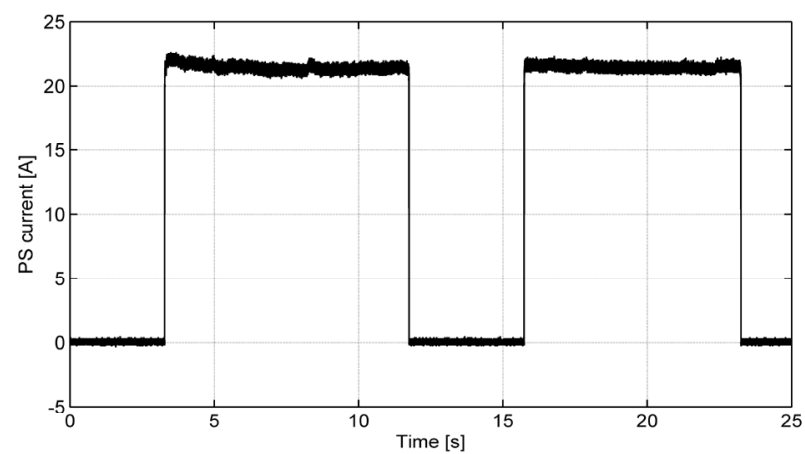

Fig. 2. Example of Power Supply current showing visible polarization in the first pulse.
The method is easy and quick and, although some precautions should be applied for a correct measurement [2], its use, when possible, is suggested.

\subsubsection{Local conductance per length for track sections without civil structures}

In case of continuous track/rail sections longer than $2 \mathrm{~km}$, the standard suggests a "local" conductance measurement. This method is based on field distribution in soil and accurate soil resistivity measurements (see sec. 2.5.3); it needs running vehicles as a source of rail voltage and gives for free an estimate of the average rail voltage to use for EN 50122-2 conductance limit.

The test setup is shown in Fig. 3. The rail voltage $\left(V_{\mathrm{RE}}\right)$ and the difference of potential due to the electric field in the soil $\left(\mathrm{V}_{12}\right.$, between two electrodes driven into the soil) are recorded simultaneously and plotted in a graph. The slope of the linear regression of the function $\mathrm{V}_{12}\left(\mathrm{~V}_{\mathrm{RE}}\right)$, named "stray current transfer ratio", is used to estimate track-to-earth conductance.

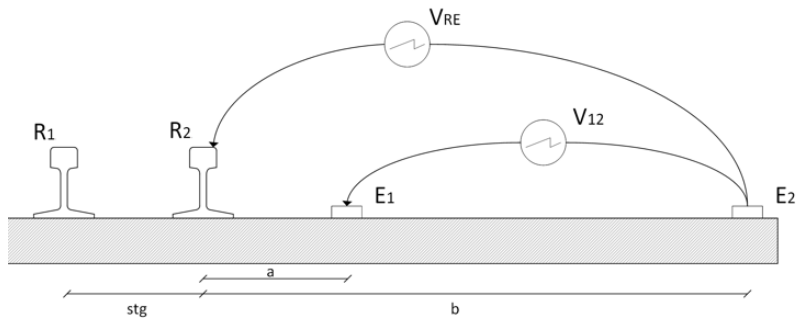

Fig. 3. Local track to earth conductance measurement using EN 50122-2 A.4 method.

The track-to-earth conductance is given by:

$$
\begin{gathered}
G_{s t}=\frac{m_{s r} 2000 \pi}{\rho\left[\frac{\ln \left(b\left(b+s_{t g}\right)\right)}{\ln \left(a\left(a+s_{t g}\right)\right)}\right]} \\
G_{d t}=\frac{m_{s r} 1000 \pi}{\rho\left[\frac{\ln \left(\left(b+0.5 s_{t g}\right)\left(b+0.5 s_{t g}+s_{t d}\right)\right)}{\ln \left(\left(a+0.5 s_{t g}\right)\left(a+0.5 s_{t g}+s_{t d}\right)\right)}\right]}
\end{gathered}
$$

where:

$G_{s t}$ is for single track configurations $[\mathrm{S} / \mathrm{km}]$;

$G_{d t}$ is the double track configurations $[\mathrm{S} / \mathrm{km}]$;

$m_{s r}$ is the stray current transfer ratio;

$\rho$ is the soil resistivity between $E_{1}$ and $E_{2}$ electrodes $[\Omega \mathrm{m}]$;

$a$ is the distance between external rail $\mathrm{R}_{2}$ and $\mathrm{E}_{1}[\mathrm{~m}]$;

$b$ is the distance between external rail $\mathrm{R}_{2}$ and $\mathrm{E}_{2}[\mathrm{~m}]$;

$s_{t g}$ is the track gauge [m];

$s_{t d}$ is the distance between track centres [m].

Running vehicles shall be considered ensuring reliable and stable connections to the rail. Wire fastening is also important when crossing public areas, such as streets with traffic of pedestrians, bicycles, cars, heavy vehicles. Last, measurement of soil resistivity between $E_{1}$ and $E_{2}$ shall be accurate (see e.g. sec. 2.5.3). 


\subsubsection{Conductance per length between running rails and metal reinforced structures}

This method is suitable with a continuous metallic structure along the track (inside tunnels and at viaducts), but may be applied also using soil as reference. The measured section is defined by the distance between the two external measurement points (A and B). Voltages between track and metal reinforcement are measured at three locations: A, B and PS (see Fig. 4).

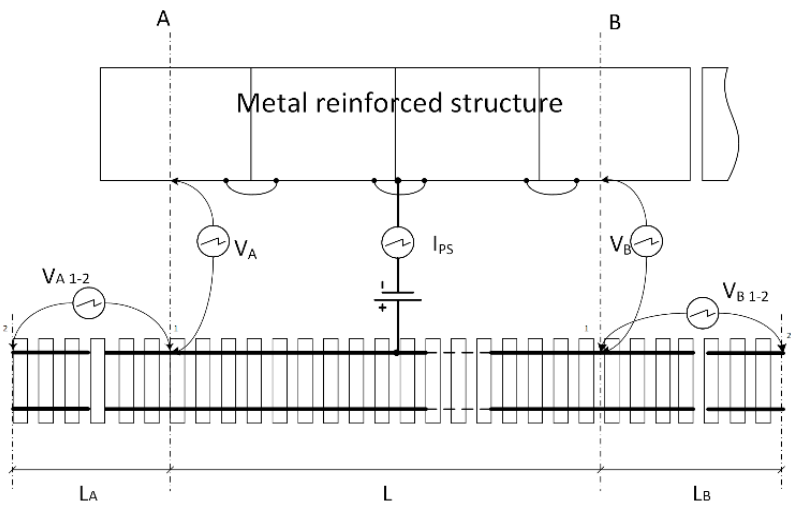

Fig. 4. Track to reinforced metallic structures conductance measurement, using EN 50122-2, A.2 method.

The rail currents flowing away from A and B points are estimated using rail voltage drop measurements and the rail resistance value (measured separately at the same location). All measurements shall be synchronized.

Track to earth conductance is calculated as follows:

$$
G=\frac{I_{P S}-\frac{V_{A 1-2}}{R_{\text {rail }}}-\frac{V_{B 1-2}}{R_{\text {rail }}}}{L \frac{V_{A}+V_{B}+V_{P S}}{3}}
$$

where:

$G$ is the track to earth conductance $[\mathrm{S} / \mathrm{km}]$;

$I_{P S}$ is the power supply current [A];

$V_{A 1-2}$ is the potential difference caused by the current leaving the section across measurement point A [A];

$V_{B 1-2}$ is the potential difference caused by the current leaving the section across measurement point $\mathrm{B}[\mathrm{A}]$; $R_{\text {rail }}$ is the rail resistance measured at the same points where $\mathrm{V}_{\mathrm{A}}$ and $\mathrm{V}_{\mathrm{B}}$ will be measured $[\Omega / \mathrm{km}]$

$V_{A}, V_{B}$ and $V_{P S}$ are the voltage between the rail and the structure at points A, B and at power supply PS (voltage $\mathrm{V}_{\mathrm{X}}=\mathrm{V}_{\mathrm{X} \text { on }}-\mathrm{V}_{\mathrm{X} \text { off, }}$, difference between on and off state readings) [V];

$L$ is the distance between A and B $[\mathrm{km}]$.

The application of this method is not straightforward. Readings shall be synchronized between measurement locations that may be far away. Length $L$ cannot be chosen arbitrarily large (so to reduce the overall number of measurements over the whole line): site experience discourages this choice. The factors to keep under visual control are several, especially when the measurement is performed during construction phases. Equipment and service boogies outside the measured track section are connected to the rail for safety reasons, thus driving into it any leaking ground current, or, worse, connecting the rail to the earth or tunnel structure while operating. Activities affecting the electric status of the track shall be suspended, but coordination between different companies and contractors is always a hard task. Easy communication between operators shall also be ensured. Usually 500 to $800 \mathrm{~m}$ section length is adequate.

Mains and high frequency noise is often unavoidable, making post-processing more complicated and impeding accurate interpretation in place (as a check for the need of repeating the measurement). When measurement positions are close to rail cuts or insulating rail joints noise is usually larger. In general, a hardware low-pass filter is beneficial, also as an anti-aliasing measure. Averaging by software may be ineffective and too dependant on averaging window length, if noise is not stationary and features large fluctuations.

For the estimation of $\mathrm{I}_{\mathrm{A}}$ and $\mathrm{I}_{\mathrm{B}}$ currents rail resistance measurement shall be accurate, including variations due to temperature changes, and the effect of rail welding (see sec. 2.5.1). The best solution would be measuring the two $I_{A}$ and $I_{B}$ currents without the need to calculate them as the ratio of voltage drop at $A$ and $B$ and estimated rail resistance, e.g. using bridge methods or $\mathrm{dc}$ current probes fit around the rail.

In general, care must be used for long tracks, where PS current may be very large and unbalanced between A and B sides. Reversing PS polarity may be useful to avoid polarization issues and to check repeatability, although less effective than for method in sec. 2.1.1.

\subsection{ASTM G165}

ASTM standard G165 [4] reports a track-to-earth conductance measurement method, based on empirical considerations. The method applies to rails and turnouts sectioned by simple rail cuts or IRJs. The maximum track length measurable is $2750 \mathrm{~m}$, the minimum $60 \mathrm{~m}$.

A minimum of three measurements per track section is required against operator's errors and noise: the highest and lowest measured values shall not alter the average of the measurement by more than $3 \%$.

The measurement set up is shown in Fig. 5: the power supply positive pole is connected to the track and the negative pole to an earthing electrode with a resistance to earth lower than $1 / 100$ of the expected track-to-earth resistance (for a track of $1 \mathrm{~km}$ the required earthing resistance shall be a fraction of $\Omega$ ). Voltage measurements are performed using ground and the track behind the measured section as voltage reference.

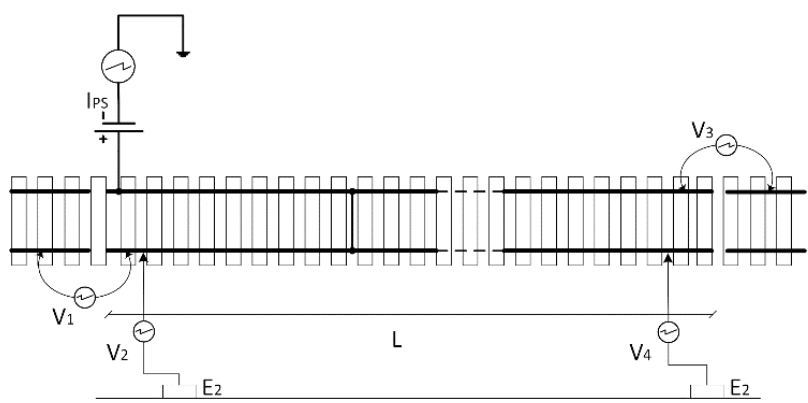

Fig. 5. Track to earth conductance measurement using ASTM G165-99 method. 
Track to earth conductance $G_{n}$ is obtained for each measurement point as the ratio of the current of the power supply and the measured voltage:

$$
G_{n}=\frac{I_{P S}}{V_{n}}
$$

where:

$G_{n}$ is the conductance of the n-th measurement point $[\mathrm{S}]$;

$I_{P S}$ is the power supply current [A];

$V_{n}$ is the voltage at the n-th measurement point [V].

The method is very similar to sec. 2.1.1, with several voltage readings. $\mathrm{G}_{3}$ and $\mathrm{G}_{2}$ are used as a check of the correct insulation of the measured track section, while $\mathrm{G}_{1}$ and $G_{4}$ are the real track-to-earth conductance values $\left(G_{4}\right.$ is the best measuring point). If a large variation of measurement results is observed, too a large PS earthing resistance may be suspected.

This method requires a large number of simultaneous voltage readings and it is thus rarely used. It brings "for free" joint efficiency when measuring $\mathrm{G}_{3}$ and $\mathrm{G}_{2}$, that are required to vary less than $3 \%$ for a well-insulated track.

\subsection{Close Potential survey}

This technique is derived from cathodic protection system investigations [5] and, although not commonly used, it may be useful if local "hot points" of track to earth conductance, as caused by defects in the rail insulation, are suspected. The method is an extension of the one in sec. 2.1.1 using many voltage readings along the measured track section. In cathodic protection surveys voltage measurements are relieved from the necessity of synchronization, but for track-to-earth conductance estimation this practice is highly discouraged: voltage readings shall be simultaneous and synchronized with power supply current reading.

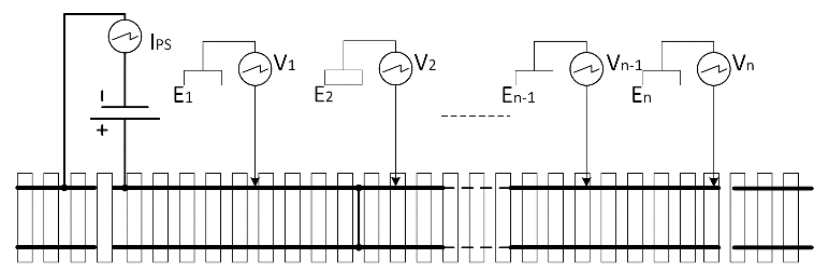

L

Fig. 6. Track to earth conductance measurement using close potential survey method.

The track-to-earth conductance is calculated as in sec. 2.1.1, taking the ratio of PS current and voltage reading; the same approximations and limitations apply. Significant variations between adjacent voltage readings indicate a local defect with a higher local track-to-earth conductance value; the operator shall be aware that going towards the end of the section voltage amplitude naturally decreases [2], so that attention shall be given to abrupt variations of voltage and not to a degrading voltage when moving toward section ends.

\subsection{Resistance to earth method}

This method is the application of the commonly used method to measure earth resistance of an earthing mesh in civil buildings: the farthest electrode $\mathrm{E}_{2}$ applies the test current; a second electrode closer to the rail is used to read the rail potential. This method can be applied only to insulated track sections delimited by rail cuts or IRJs. The length $L$ of the section shall be measured.

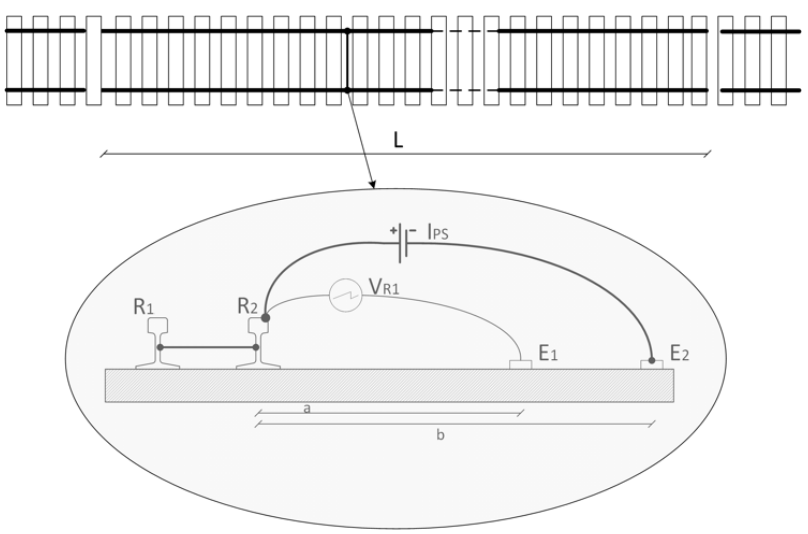

Fig. 7. Track to earth conductance measurement using resistance to earth method.

The voltage between the track and a probe electrode E1 is measured and the track-to-earth conductance is given by:

$$
G=\frac{I_{P S}}{L V_{R 2}}
$$

where:

$G$ is the track-to-earth conductance $[\mathrm{S} / \mathrm{km}]$;

$\mathrm{I}_{\mathrm{PS}}$ is the power supply current $[\mathrm{A}]$;

$V_{R 2}$ is the voltage between $R_{2}$ (electrically bonded to

$\mathrm{R}_{1}$ ) and probe electrode $\mathrm{E}_{1}[\mathrm{~V}]$;

$a$ is the distance between $\mathrm{R}_{2}$ and voltage electrode $\mathrm{E}_{1}$ [m];

$b$ is the distance between $\mathrm{R}_{2}$ and current electrode $\mathrm{E}_{2}$ $[\mathrm{m}]$;

$L$ is the section length $[\mathrm{km}]$.

Care shall be paid in placing the two electrodes as they shall not be influenced by the track electric field. The most widespread rule to place electrodes is the $62 \%$ rule $(a=0.62 b)$; this choice may be led back to the $62 \%$ rule for flatness of potential curve of the Fall-ofPotential method, described in [6], that, however, is strictly valid in homogeneous soil conditions.

The method is very fast and useful as preliminary test to save time later during measurements. It can be performed with a common earth resistivity tester, taking care that the resulting conductance be in the measurement range of the instrument.

\subsection{Auxiliary measurements}

As mentioned in previous sections, several methods need an estimate of some system parameters, such as rail resistance or soil resistivity. They are reviewed in the following. 


\subsubsection{Rail resistance}

The measurement of rail resistance is of fundamental importance for methods described in sec. 2.1.3. The measurement method described here is that suggested by EN 50122-2, Annex A.1 (see Fig. 8).

A track section delimited by rail cut or insulating rail joints is used. The two rails of the track are bonded together at section ends and a current is applied in the center of the 8-shape loop circuit formed by the two bonded rails. In case of electrically continuous track, the two bonding connections alleviate the problem and can define quite effectively the track section under measurement if well bonded to the rails and of low resistance. The voltage drop across two adjacent rail sections on the same rail caused by the flowing current is measured using two voltmetric readings for a given length, $\mathrm{L}_{1}$ and $\mathrm{L}_{2}$, that shall be measured accurately.

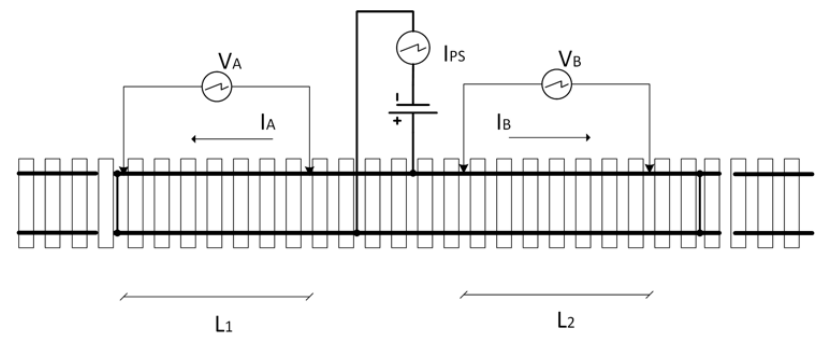

Fig. 8. Rail resistance measurement (EN 50122-2, A.1).

The resistance of the rail is calculated as follows:

$$
R_{\text {rail }}=\frac{V_{A}+V_{B}}{\left(L_{1}+L_{2}\right) I_{P S}}
$$

where:

$R_{\text {rail }}$ is the rail resistance [ $\Omega$;

$I_{P S}$ is the current of the power supply [A];

$L_{1}$ and $L_{2}$ are the rail lengths for $\mathrm{V}_{\mathrm{A}}$ and $\mathrm{V}_{\mathrm{B}}$ voltage drop measurements [km];

$\mathrm{V}_{\mathrm{A}}$ is the $\mathrm{V}_{\text {on }}-\mathrm{V}_{\text {off }}$ voltage drop in sec. A of length $\mathrm{L}_{1}$ [V];

$V_{B}$ is the $V_{\text {on }}-V_{\text {off }}$ voltage drop in sec. B of length $L_{2}$ [V].

Repeated measurements are advisable to evaluate consistency and uncertainty. However, such approach is ineffective for systematic errors: the two lengths shall be measured more than once, also to exclude operator's mistakes; rail temperature influences significantly rail resistivity [7]; rail welds are quite relevant, as they are allowed to increase overall rail resistance up to $5 \%$, so that measuring points shall correspond to those used for the voltage drop measurement of the track insulation test. It is reminded that the so obtained rail resistance value is used to estimate the current flowing from voltage drop measurements; the difference at the numerator of eq. (4) may be quite small for good track insulation and small errors in the estimate of " $R_{\text {rail }}$ " are thus amplified.

Current intensity may be very large (e.g. hundred Amps for just $12 \mathrm{~V}$ PS) and voltage drops along very short rail and wire lengths already significant: 4 wires techniques shall be adopted and galvanic isolation between channels is a must.

\subsubsection{Remote electrode distance}

This section describes a method derived from cathodic protection measurements [5] to find out the correct distance for placing the electrode to consider it electrically remote from the track.

A track section delimited by insulating rail joints or rail cuts is considered, but the method can be applied also to electrically continuous tracks. PS is connected between track and ground reference at the beginning of the section; at the other end of the section (chosen to avoid influence from PS earthing electrode), the track voltage is measured with respect to a movable electrode driven into the soil at various distances from the track.

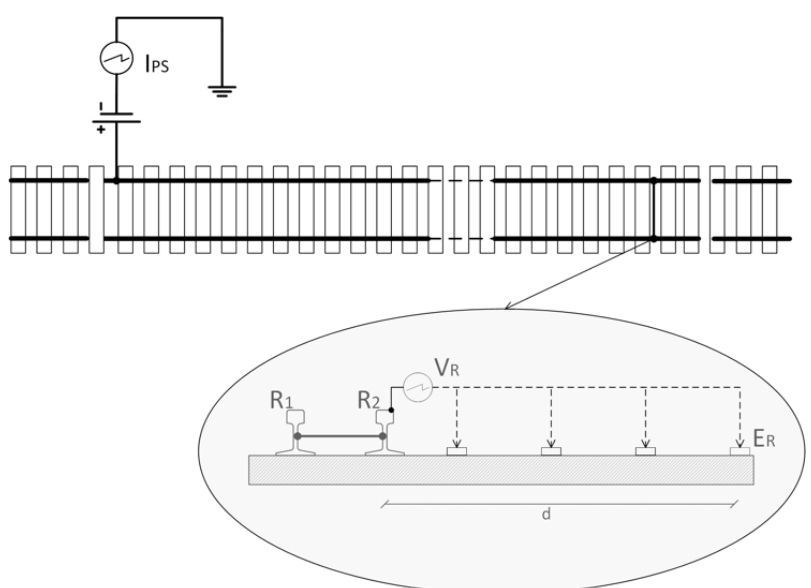

Fig. 9. Test set-up for the determination of the electrically remote electrode position.

The voltage $V_{R}$ follows a logarithmic decay vs. distance from track and then finally reaches a constant value, corresponding to the minimum distance so to consider the electrode electrically remote. As for other methods where flatness of potential curve is to be evaluated (e.g. Fall-of-Potential method), a suitable criterion for flatness is needed: e.g. the spread of three adjacent points is $<5 \%$ of the average, where minimum increment of distance is $10-20 \%$ at each point. Plotting $V_{R}$ vs. distance is of great help to interpret results.

\subsubsection{Soil Resistivity}

Soil resistivity may be measured by driving four electrodes into the soil and using a soil resistivity meter implementing Wenner or Schlumberger methods [6][8].

\section{Practical tips}

Some practical tips may be formulated based on previous considerations and experience.

For measurements inside tunnels, mobility is extremely important, since access to the track during construction may be from a few entry points (e.g. at some stations), requiring long walks to reach the desired location. Measuring equipment should fit into backpacks and trolleys avoiding need of bogies.

If reinforcement is not electrically continuous (e.g. when tunnel sections are gapped or prefabricated tunnel 
lining segments are used), electrically continuous and earthed cable trays may be used as reference.

Communication inside tunnels may be difficult due to fan noise, on-going activities and poor radio propagation.

When measuring in urban areas, a good earth reference may be hard to find for the soil cannot be reached due to asphalt and constructions: green areas with deep soil shall be considered (flowerbeds are made by landfill and are not a good earth reference). Concrete electrodes of the $\mathrm{CuSO}_{4}$ type may be also considered as reference for voltmetric readings, but the effect of buried pipes and cables shall be verified, e.g. by moving the electrodes to check variability.

Wires connecting to electrodes shall cross the road, and will be damaged and may also cause accidents if not fastened and protected (e.g. by heavy tape glued to the asphalt). High visibility tape should always be used to indicate the presence of wires, including pedestrian and green areas, useful also in case of grass cut.

Connection to rails shall be effective both for mechanical robustness, contact resistance and rapidity to connect and disconnect: there are rail clamps for this purpose, although not all ensure the necessary mechanical stability and that rust is removed when inserted; for rails embedded in concrete or soil the accessible part is the top of rail, for which modified bench clamps or naked conductor pressed against the rail surface may be used.

Standards are not clear if track insulation is to be evaluated also in wet conditions. The correct mix of dry and wet conditions representative of weather conditions for a specific location shall be considered, when comparing to the limits. In all cases, measurements should be done when a suitable time interval has elapsed after track wetting to let surface dry, avoiding the measurement of the conductance of water itself.

When using rail cuts to isolate the track section, rail length may change significantly if temperature varies due to e.g. the position of the sun: isolation may be compromised, also if rubber sheets were interposed, because the applied force is extremely intense.

As an alternative to rail current measurement through voltage drop, current clamps may be used for a direct measurement: clamps with such a large section are all of the Rogowski type and cannot measure dc, so that a suitably low frequency signal shall be used, that can reasonably replace dc (artefacts may be observed when the ac signal changes its polarity at each half cycle due to soil polarization).

After data of a measurement have been recorded, a preliminary in-place calculation of relevant quantities and evaluation of measurement quality is nevertheless important to decide if the measurement shall be repeated and to avoid useless complications in post-processing.

\section{Conclusions}

Several methods have been reviewed for the measurement of track-to-earth conductance. For all uncertainty is caused not only by instrumental uncertainty, but also variability of system parameters, including soil and rail resistivity. Repeatability is suggested as a means to monitor measurement accuracy. Outliers and aberrations can always occur due to external factors, mostly practical, such as unavoidable working activities and unnoticed earthing of the rails under measurement (e.g. by OVLDs, negative feeder cables, accidental contact with earthed conductive parts).

An electrically insulated track section is always the best configuration for accuracy and to avoid propagation of electric noise from other parts of the line. Examples of continuous conductive structure are several: tunnel structure, walkways, handrails and cable trays, or a longitudinal earthed conductor.

Methods in sec. 2.1.1 and 2.1.3 are quite accurate without and with such conductive structures. Increasing the number of voltmetric readings brings to the method in sec. 2.3, useful to spot out local defects of insulation. An equivalent method based on the difference of measured rail current along the track section is currently under study, using e.g. mentioned Rogowski coils.

For minimum impact on an existing line with running trains method in sec. 2.1.2 is almost a forced choice: thanks to the averaging of several points, the method is quite accurate and copes with sporadic transients; the worst aspect is the lack of a literature reference to determine the extension of the measured section at the left and right ends of the voltmetric terminal.

\section{References}

1. EN 50122-2 / IEC 62128-2, Railway applications Fixed installations -Electrical safety, earthing and the return circuit - Part 2: Provisions against the effects of stray currents caused by dc traction systems (CENELEC, Brussels, Belgium, 2012)

2. J. Bongiorno and A. Mariscotti, Accuracy of railway track conductance and joint efficiency measurement methods, Acta IMEKO, 4, 82-87 (2015).

3. J. Bongiorno and A. Mariscotti, Variability of track to ground conductance measurement, 22th IMEKO TC4 Int. Symp. proceedings, Iasi, 14-15 Sep. 2018.

4. ASTM G165-99, Standard Practice for Determining Rail-to-Earth Resistance, ASTM International, Conshohocken, Pennsylvania, United States (2005)

5. A.W. Peabody, Control of pipeline corrosion. $2^{\text {nd }}$ ed., (NACE International, Houston, 2001)

6. IEEE Std. 80, IEEE Guide for Safety in $A C$ Substation Grounding, Piscataway, New Jersey, United States (2013)

7. A. Mariscotti and P. Pozzobon, Resistance and Internal Inductance of traction rails: a survey, IEEE Trans. on Vehicular Technology, 53, 1069-1075 (2004).

8. A. Samouelian, I. Cousin, A. Tabbagh, A. Bruand and G. Richard, Electrical resistivity survey in soil science: a review, Soil and Tillage Research, 83, 173-193 (2005) 